\title{
Segmental Relaxation in End-Linked Poly(dimethylsiloxane) Networks
}

\author{
M. J . Schroeder \\ Department of Chemistry, United States Naval Academy, Annapolis, Maryland 21402
}

\section{M. Roland*}

Chemistry Division, Code 6120, Naval Research Laboratory, Washington, D.C. 20375-5342

Received September 25, 2001

\begin{abstract}
End-linked polydimethysiloxane (PDMS) networks were prepared by end-linking vinylterminated PDMS chains with tetrafunctional silanes. Dielectric $\alpha$-relaxation spectra, thermal expansivities, and the (rubbery) elastic modulus were measured for these materials. From the cross-link density at which marked changes occur in the segmental relaxation times and the $\mathrm{T}_{\alpha}$-scaled temperature dependence (fragility), a characteristic length for the $\alpha$-relaxation was determined. The result, $14 \AA$ at $\mathrm{T}_{\alpha}$, is quite close to the length scale associated with constraint of crystallization in similar PDMS networks. Cross-linking also affects the thermal expansion and elastic modulus, although these properties exhibit a smoother variation with cross-link density.
\end{abstract}

\section{Introduction}

Relaxation near the glass temperature ( $\alpha$-relaxation) in polymers and small molecule glass formers underlies myriad phenomena of current interest, such as finite size effects, ${ }^{1,2}$ dynamic heterogeneity, ${ }^{3-5}$ the J ohariGoldstein process, ${ }^{6,7}$ the excess wing in the $\alpha$-dispersion, ${ }^{8,9}$ and the constant loss. ${ }^{10-12} \mathrm{~A}$ fruitful approach to investigating local segmental relaxation is to chemically alter the material in order to develop structure/ property relationships. One such change that strongly affects the properties of the $\alpha$-relaxation is cross-linking. Restrictions imposed by the network junctions reduce the configurational freedom of the chains, increasing the local friction as well as enhancing intermolecular cooperativity. ${ }^{13-16}$ The results are a depression of the glass temperature and segmental relaxation times which become both longer and more sensitive to temperature.

In this paper we describe a study of the effect of crosslinking on the segmental relaxation of end-linked poly(dimethylsiloxane). The expectation is that when the network chain length becomes small, relative to the length scale associated with the local segmental dynamics, strong cooperativity effects will become manifest. The results are compared to the effect of cross-linking on other physical properties of the network.

\section{Experimental Section}

Elastomeric networks (Table 1 ) were prepared by endlinking vinyl-terminated poly(dimethylsiloxane) (PDMS) chains with tetrafunctional silanes using well-established hydrosilylation methods. ${ }^{17-19}$ The linear PDMS, tetrakis(dimethylsiloxy)silane cross-linking agent, and the catalyst (a platinum-divinyltetramethyldisiloxane complex) were obtained from Gelest, Inc. The silane and PDMS were mixed in the stoichiometric ratio of 1.4:1 (active hydrogen atoms, $\mathrm{SiH}$, to chain vinyl groups). A $2.3 \%$ solution of the catalyst in xylene was diluted 10-fold with HPLC-grade toluene and added to achieve not less than $30 \mathrm{ppm}$ Pt in the final mixture. After thorough mixing, samples were poured into aluminum molds, degassed under vacuum, and allowed to air-dry overnight. To ensure complete reaction, postcuring was carried out at $80^{\circ} \mathrm{C}$ for $48 \mathrm{~h}$ in vacuo. N etworks for dielectric measurements were
Table 1. PDMS End-Linked Networks

\begin{tabular}{ccccl}
\hline$M_{\mathrm{c}}(\mathrm{g} / \mathrm{mol})$ & $\mathrm{N}$ & $\left\langle\mathrm{r}^{2}\right\rangle^{1 / 2} \mathrm{a}(\AA)$ & $\alpha\left(\mathrm{deg}^{-1}\right)$ & $\mathrm{E}(\mathrm{Pa})$ \\
\hline linear & & & & 0 \\
62700 & 1700 & 170 & $2.62 \times 10^{-4}$ & $8.2 \times 10^{4}$ \\
28000 & 760 & 110 & $2.75 \times 10^{-4}$ & $2.8 \times 10^{5}$ \\
17200 & 460 & 90 & $2.73 \times 10^{-4}$ & $6.9 \times 10^{5}$ \\
9400 & 260 & 66 & $2.25 \times 10^{-4}$ & $8.7 \times 10^{5}$ \\
6000 & 162 & 53 & $1.92 \times 10^{-4}$ & \\
770 & 20 & 18 & $1.65 \times 10^{-4}$ & $1.8 \times 10^{6}$ \\
500 & 13.6 & 14 & $1.27 \times 10^{-4}$ & $2.0 \times 10^{6}$ \\
186 & 5 & 8 & $1.0_{2} \times 10^{-4}$ & $1.0 \times 10^{7}$
\end{tabular}

a From eq 5 using characteristic ratio taken from ref 33.

extracted in toluene-methanol for 4 days, deswelled in methanol, and then dried overnight at $80^{\circ} \mathrm{C}$ under vacuum. The extractable content of all networks was less than $5 \mathrm{wt} \%$. Samples used for thermal analysis and mechanical tests were not extracted. All samples were stored in a desiccator prior to use.

I sothermal dielectric measurements were obtained using a time domain spectrometer (Imass Inc.) having a nominal frequency range $-3.2<\log \omega(\mathrm{rad} / \mathrm{s})<4.8$. A $25 \mathrm{~mm}$ parallel plate geometry was employed, with a guard ring on the detector side and an electrode separation between 0.05 and $0.2 \mathrm{~mm}$. A Delta Design model 9023 oven provided temperature control to $\pm 0.1 \mathrm{~K}$. To prevent arystallization, samples were quenched ( $>50 \mathrm{~K} / \mathrm{min}$ ) with a stream of liquid nitrogen to low temperature and then allowed to equilibrate for at least an hour after warming to the desired temperature. Replicate measurements were obtained to ensure an absence of crystallization.

Thermal expansion in the rubbery state was measured with a Perkin-EImer TMA-7. Samples 4-7 mm thick were obtained by stacking. The probe tip applied a $2 \mathrm{mN}$ force on the sample. Thermal cycling was done between 20 and $-140{ }^{\circ} \mathrm{C}$ at a rate of $5{ }^{\circ} \mathrm{C} / \mathrm{min}$. The linear thermal expansion coefficient at $0{ }^{\circ} \mathrm{C}$ was determined from the sl ope and intercept of the expansion vs temperature, in the region above the crystalline melting point.

Equilibrium stress-strain curves at $25^{\circ} \mathrm{C}$ were obtained with a Lloyd Instruments tester, using dumbbell-shaped samples ( $38 \mathrm{~mm}$ long $\times 5 \mathrm{~mm}$ wide $\times 1.5 \mathrm{~mm}$ thick). Samples were slowly extended stepwise, with the intermittent strain maintained until mechanical equilibrium was established. The modulus was obtained from the initial, linear portion of the stress/strain response. 


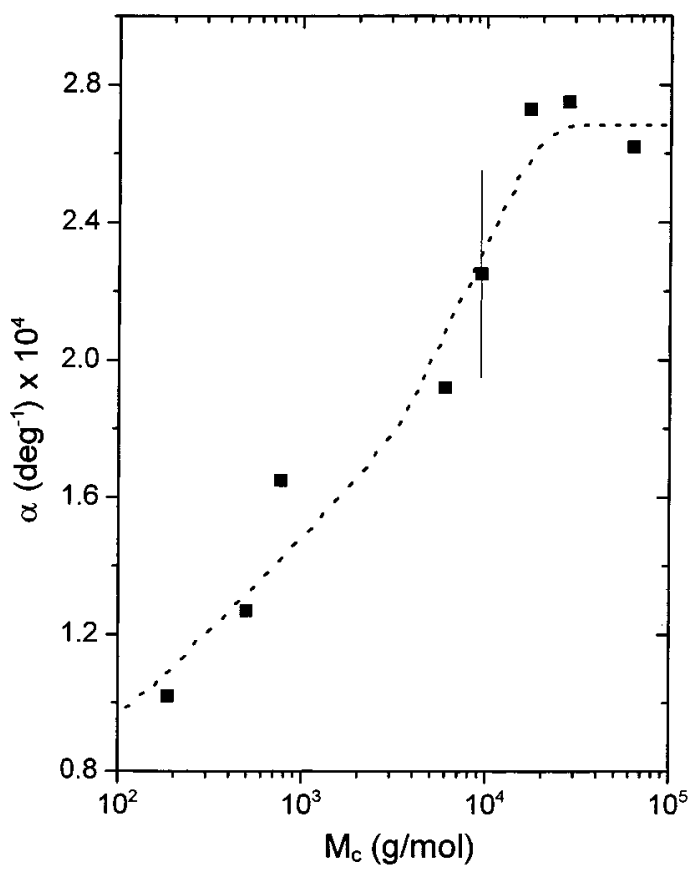

Figure 1. Linear thermal expansion coefficient for the PDMS networks. The error bar denotes the limit of error. The dotted line is only to guide the eyes.

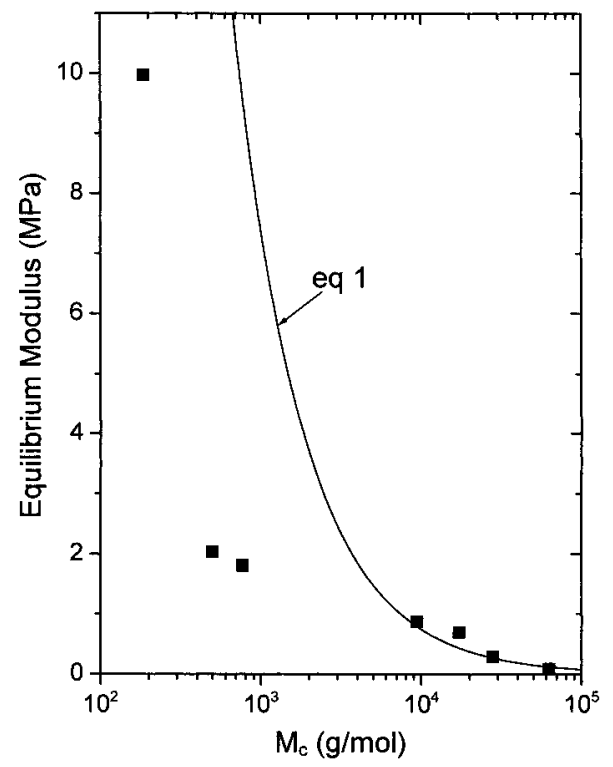

Figure 2. Tensile modulus measured at mechanical equilibrium as a function of the molecular weight between cross-links. The solid line is eq 1, using the precursor molecular weights for $M_{c}$ (i.e., no adjustable parameters).

\section{Results}

Thermal Expansivity. Volume contraction upon cooling of a polymer above $T_{g}$ reflects the loss of free volume. Thermal expansion coefficients measured for the PDMS networks in the rubbery amorphous state are displayed in Figure 1. At low degrees of cross-linking, the behavior is identical to linear PDMS. This changes, however, when the molecular weight between crosslinks, $M_{c}$, falls bel ow ca. $10^{4} \mathrm{~g} / \mathrm{mol}$. F or the more crosslinked networks, the expansion coefficient becomes a monotonically decreasing function of cross-link density.

Elastic Modulus. In Figure 2 we plot the equilibrium Young's modulus, measured at strains ( $<40 \%)$ for which the stress was proportional to the strain. Since at these

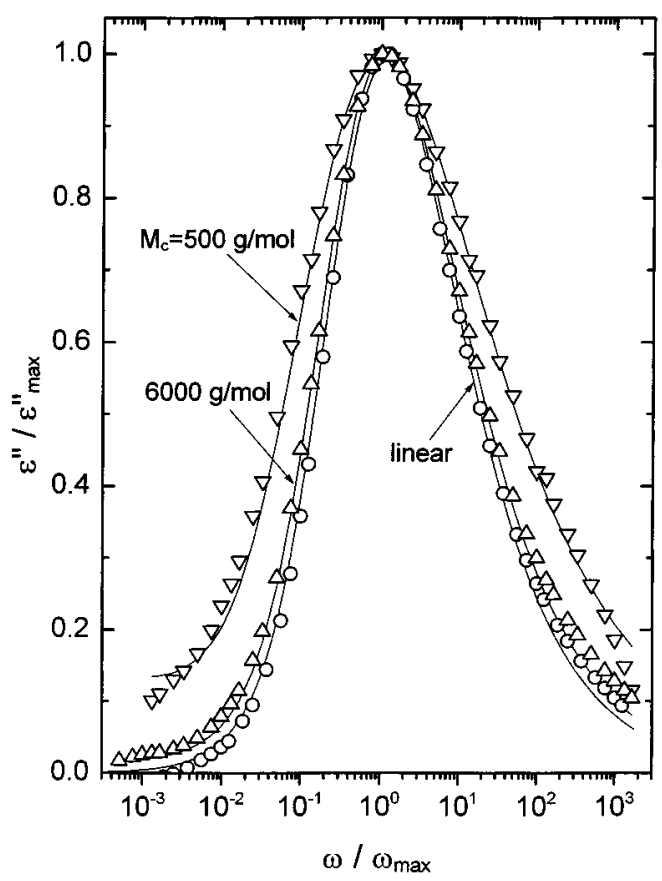

Figure 3. Dielectric loss peaks for the linear PDMS and two networks, normalized to superimpose at the respective maxima. The solid lines are fits to eq 2.

lower strains the effect of intermolecular constraints is greatest, we use the affine model of rubber elasticity to describe the modulus. ${ }^{20,21}$

$$
\mathrm{E}=3 \rho \mathrm{RT} / \mathrm{M}_{\mathrm{c}}
$$

where the mass density, $\rho$, is taken to be $1 \mathrm{~g} / \mathrm{cm}^{3}$ (although actually it is cross-link density dependent ${ }^{22}$ ), and RT has its usual significance. The curve cal culated from eq 1 is included in Figure 2. Note that there are no adjustable parameters, since the molecular weight between cross-links is just the molecular weight of the precursor chains.

Segmental Relaxation. Representative dielectric loss spectra are shown in Figure 3. The $\alpha$-dispersion systematically broadens with increasing cross-link density (Figure 4). This has been reported previously for other polymer networks. ${ }^{14,23}$ With the exception of the most cross-linked PDMS, the peaks could be fit to the transform of the Kohlrausch function ${ }^{24}$

$$
\epsilon^{\prime \prime}=\Delta \epsilon \exp \left\{-\left(\mathrm{t} / \tau_{\mathrm{K}}\right)^{\beta}\right\}
$$

in which $\Delta \epsilon$ is the dielectric strength and $\tau_{\mathrm{K}}$ a relaxation time. The values obtained for the stretch exponent $(\beta$ in Table 2) reflect the peak breadths. For the network with the highest level of cross-linking $\left(M_{c}=186 \mathrm{~g} / \mathrm{mol}\right)$, the dispersion is extremely broad (Figure 4) and lacks the asymmetry toward higher frequency of the Kohlrausch equation. This sample is also the only network exhibiting any marked dependence of its peak shape on temperature, the breadth of the dispersion increasing roughly $20 \%$ for a 6 deg temperature decrease.

Since the $\alpha$-relaxation for $M_{c}=186 \mathrm{~g} / \mathrm{mol}$ does not conform to eq 2, we use the frequency of the maximum in the loss peak to define a most probable relaxation time, $\tau_{\alpha}=1 / 2 \pi \mathrm{f}_{\mathrm{p}}$. This relaxation time is longer than the $\tau_{\mathrm{K}}$ obtained by fitting the spectra to eq 2 (by about 35\% for $\beta=0.5$ ). However, since for all networks except the most cross-linked $\beta$ is invariant to temperature, $\tau_{\alpha}$ and 


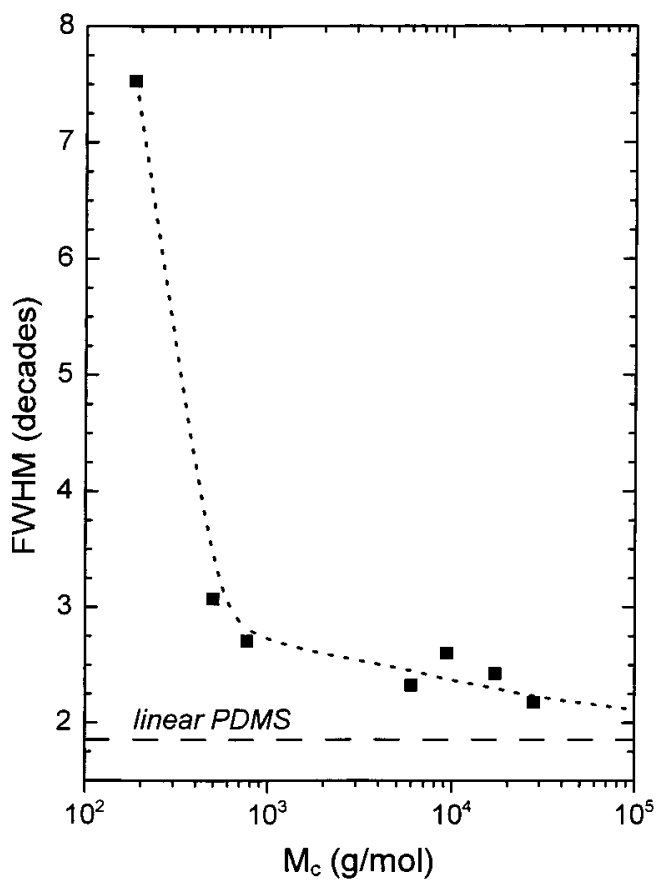

Figure 4. Network chain length dependence of the full width at high maximum of the $\alpha$-dispersion in the dielectric loss. The peak shape was independent of temperature, except for $\mathrm{M}_{\mathrm{c}}=$ $186 \mathrm{~g} / \mathrm{mol}$. For this network, the fwhm increased by almost 0.3 decade/deg (the value in the figure corresponds to $205 \mathrm{~K}$ ). The dashed line represents the breadth of un-cross-linked PDMS, while the dotted line is only to guide the eyes. For a Debye peak ( $\alpha=1$ in eq 1 ), fwhm $=1.14$ decades.

Table 2. Segmental Relaxation Properties

\begin{tabular}{cccccc}
\hline $\mathrm{M}_{\mathrm{c}}(\mathrm{g} / \mathrm{mol})$ & $\mathrm{T}_{\alpha}(\mathrm{K})$ & $\beta($ eq 2$)$ & $\log \mathrm{a}(\mathrm{s})$ & $\mathrm{b}(\mathrm{K})$ & $\mathrm{T}_{0}(\mathrm{~K})$ \\
\hline linear & 148.0 & 0.51 & -18.51 & 1105 & 122.1 \\
28000 & 148.4 & 0.52 & -13.48 & 517.1 & 131.8 \\
17200 & 148.6 & 0.50 & -12.40 & 447.8 & 132.9 \\
9400 & 149.2 & 0.43 & -11.49 & 386.4 & 134.5 \\
6000 & 149.4 & 0.42 & -13.58 & 539.3 & 132.2 \\
770 & 154.0 & 0.43 & -14.69 & 673.9 & 134.1 \\
500 & 157.2 & 0.37 & -11.47 & 402.1 & 142.0 \\
186 & 202.4 & & -10.60 & 271.9 & 191.3
\end{tabular}

$\tau_{\mathrm{K}}$ have the same temperature dependence. The temperature dependence of $\tau_{\alpha}$ is shown in Figure 5 for several networks. The curves through the data are fits to the Vogel-Fulcher equation ${ }^{25}$

$$
\tau_{\alpha}=\mathrm{a} \exp \left(\frac{\mathrm{b}}{\mathrm{T}-\mathrm{T}_{0}}\right)
$$

with values obtained for the fitting parameters $a, b$, and $T_{0}$ listed in Table 2.

At the lower cross-link densities, there is only a modest increase in relaxation time with decreasing $M_{c}$; however, for $M_{c}<500 \mathrm{~g} / \mathrm{mol}, \tau_{\alpha}$ increases substantially. This is illustrated in Figure 6 , in which $T_{\alpha}$, the temperature at which $\tau_{\alpha}=1 \mathrm{~s}$, is plotted as a function of the molecular weight between cross-links. This curve parallels the peak breadth data in Figure 4.

It is common practice to scale segmental relaxation data by normalizing the temperature variable by the glass temperature or, more commonly, by $T_{\alpha}$. Such fragility (or cooperativity ${ }^{26}$ ) curves have been shown to be a useful means to classify segmental relaxation behavior of polymers and small mol ecule glass formers. ${ }^{27-30}$ In Figure 7 we display the $\alpha$-relaxation times for the linear PDMS and all networks as a function of $T_{\alpha} / T$.

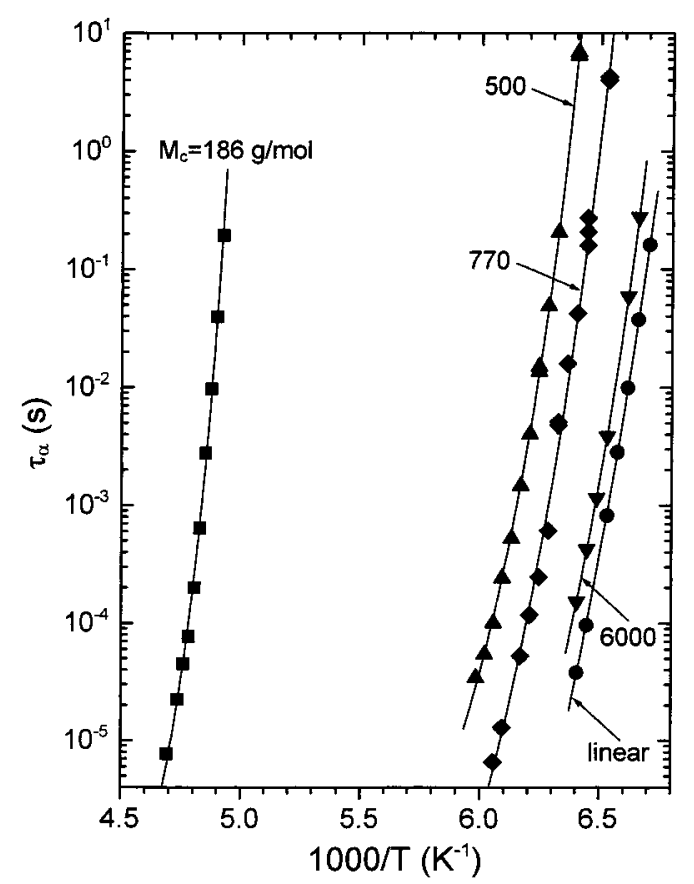

Figure 5. Local segmental relaxation times for linear PDMS and four networks along with fits to eq 3 . The molecular weight between cross-links is as indicated. The measurement range for the most cross-linked network was limited by the large breadth of its dispersion.

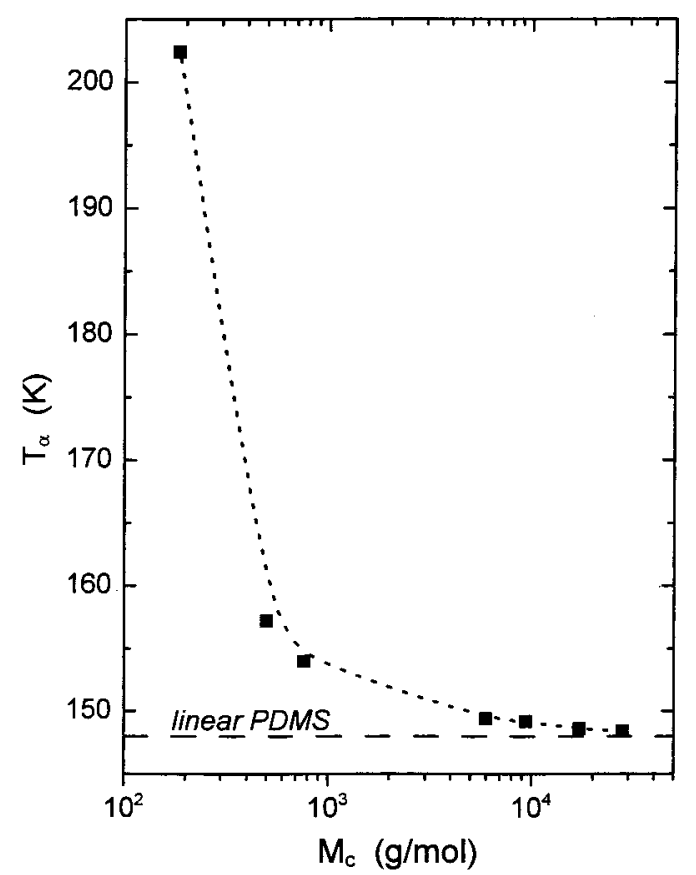

Figure 6. Dynamic glass temperature, defined as the temperature at which $\tau_{\alpha}=1 \mathrm{~s}$, for the PDMS networks. $\mathrm{T}_{\alpha}$ of uncross-linked PDMS is indicated by the horizontal dashed line.

There is no significant difference in fragility among these samples, except again for the most cross-linked $\left(\mathrm{M}_{\mathrm{c}}=186 \mathrm{~g} / \mathrm{mol}\right)$ network.

We can quantify the fragility from the slope at $T_{\alpha}$, which in terms of the Vogel-Fulcher parameters is equal to ${ }^{31}$

$$
\frac{d \log \left(\tau_{\alpha}\right)}{d\left(T_{\alpha} / T\right)}=\frac{b / T_{\alpha}}{(\ln 10)\left(1-T_{0} / T_{\alpha}\right)^{2}}
$$




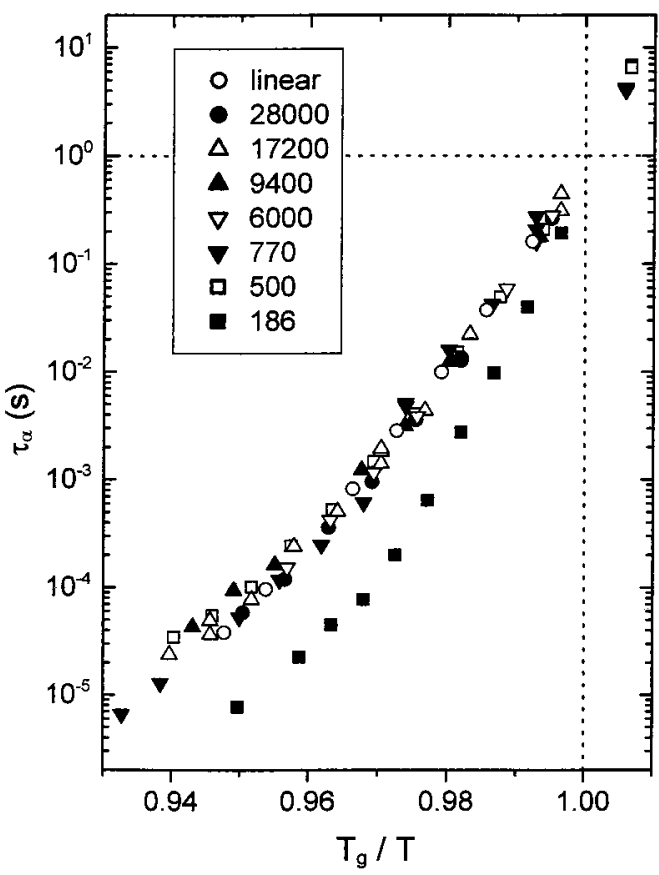

Figure 7. Fragility plots for the un-cross-linked PDMS and seven networks having the indicated molecular weight be tween cross-links. Only at the highest cross-link density $\left(M_{c}\right.$ $=186 \mathrm{~g} / \mathrm{mol}$ ) is there a significant difference in the $T_{\alpha}$ normalized temperature dependence of $\tau_{\alpha}$.

This gives a value of 106 for the linear PDMS, consistent with the literature. ${ }^{32}$ With the exception of the most cross-linked sample, the fragilities for the networks are equal to one another within experimental error and only slightly larger than for the linear PDMS. However, as seen in Figure 7, the network with $M_{c}=186 \mathrm{~g} / \mathrm{mol}$ has a much larger fragility, equal to 190 per eq 4.

\section{Discussion}

The degree of cross-linking necessary to effect a change in behavior may depend on the physical property being measured. The thermal expansivity is a bulk average of the volume expansion for all segments. For network chain lengths of the PDMS exceeding $10^{4} \mathrm{~g} / \mathrm{mol}$, the behavior is identical to linear PDMS (Figure 1). For this degree of cross-linking, less than $1 \%$ of the backbone atoms are directly bonded to a network junction. This is too sparse to influence the thermal expansion coefficient at the precision of our measurements.

At lower $M_{C}$ the expansion coefficient changes, and this can be associated with a characteristic length. The mean-square end-to-end distance is proportional to the number of backbone bonds, $\mathrm{N}$ (= $\mathrm{M} / 37$ for PDMS) as ${ }^{33}$

$$
\left\langle r^{2}\right\rangle=N I^{2} C_{n}
$$

where $\mathrm{l}$ is the bond length ( $=1.64 \AA$ for PDMS) and $\mathrm{C}_{\mathrm{n}}$ is the characteristic ratio. At sufficiently high molecular weight (>4000 $\mathrm{g} / \mathrm{mol}$ ), $\mathrm{C}_{\mathrm{n}}$ for PDMS becomes constant, 6.43. ${ }^{33}$ From the results in Figure 1 , eq 5 then gives a value of ca. $68 \AA$ for the length scale associated with volume contraction of PDMS above $\mathrm{T}_{\mathrm{g}}$. If the extent of free volume governs the rate of molecular rearrangements, ${ }^{25}$ some correspondence with the effect of crosslink density on local segmental relaxation would be expected.
Rubber elasticity models consider the effect of both the chain entropy and internal energy on the elastic energy as well as any contribution from intermolecular constraints. The latter arises from the fact that the configurations available to the network chains are constrained by intermolecular interactions, and thus such interactions can modify the stress. Although rubber el asticity considers only the response of the network at mechanical equilibrium, correspondences to the dynamic behavior of polymer melts have been shown. ${ }^{13,15}$

The proportionality in eq 1 between the modulus and $\mathrm{M}_{\mathrm{c}}{ }^{-1}$ is a universal feature of rubber elasticity models, notwithstanding differences in their predictions concerning the strain dependence of the elastic stress. Deviation from this proportionality is observed in Figure 2 around $M_{c}<4000 \mathrm{~g} / \mathrm{mol}$ (which is al so the molecular weight at which the characteristic ratio assumes its limiting value for long chains ${ }^{33}$ ). From eq 5, this mol ecular weight corresponds to a length scale $\approx 40 \AA$.

An obvious source for the broadening of the $\alpha$-relaxation peaks of the PDMS networks (Figures 3 and 4 ) is constrained motion of those segments cl ose (topol ogically or spatially) to a junction. Their contribution to the dielectric response appears at lower frequencies, in comparison to the response of chain units remote from the cross-link site. The net effect of this inhomogeneous broadening will be a shift of the observed peak toward lower frequency; however, when the cross-link density is low, the most probable relaxation time, $\tau_{\alpha}$, is not much changed.

Figure 5 reveals that when $M_{c}$ is less than ca. 500 $\mathrm{g} / \mathrm{mol}$, there is an enormous increase in the relaxation time, corresponding to a 50 deg increase in $\mathrm{T}_{\alpha}$ (Figure 6). This is accompanied by a substantial change in the network's fragility (Figure 7), along with a marked increase in the breadth of the $\alpha$-peak (Figure 4). Increased fragility reflects stronger intermolecular cooperativity. ${ }^{34} \mathrm{~F}$ or this degree of cross-linking, the network strands are sufficiently short that the characteristic ratio becomes chain length dependent. For $\mathrm{M}_{\mathrm{c}}=$ $500 \mathrm{~g} / \mathrm{mol}, \mathrm{C}_{\mathrm{n}}=5.0,35$ from which we calculate using eq 5 a length scale $\approx 14 \AA$ for the $\alpha$-relaxation at $T_{\alpha}$. This is significantly smaller than the characteristic length deduced from the thermal expansion measurements, which might be taken as an argument against free volume interpretations of segmental relaxation.

The characteristic length observed for the (dynamic) glass transition depends on temperature as well as on the experimental probe. From heat capacity spectroscopy, dimensions in the range from $10 \AA$ to as much as $35 \AA$ have been obtained. ${ }^{36} \mathrm{NMR}$ has yielded values on the order of $10 \AA .{ }^{37,38}$ Variation of the molecular weight of the components of a blend led to a characteristic length of $15 \AA .{ }^{39}$ Certainly the value obtained herein, $14 \AA$ (6-7 monomer units), is within the range of literature values, recognizing, moreover, that such a characteristic length is not a universal constant. ${ }^{40}$

To examine further the effect of cross-linking on local segmental relaxation, we use a relation from the coupling model. ${ }^{41,42}$ According to the latter, the observed $\alpha$-relaxation time is related to a noncooperative relaxation time, $\tau_{0}$, describing the putative dynamics in the absence of any intermolecular constraints. $T_{\alpha}$-normalized Arrhenius plots of $\tau_{0}$ should be quite similar for the networks, since the chain units are identical, once the effect of cooperativity arising from the network structure is removed. ${ }^{14}$ The two relaxation times 


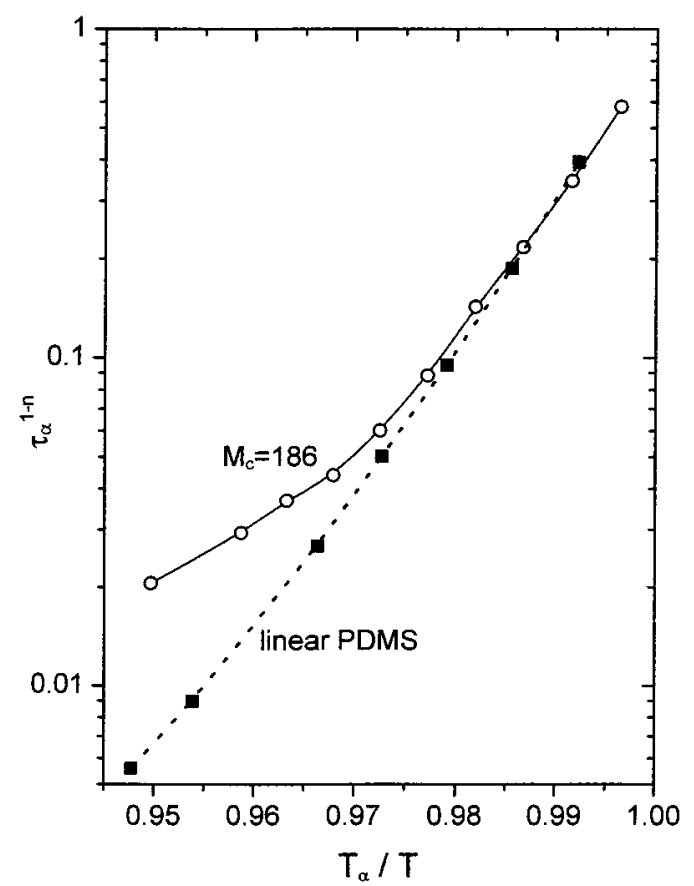

Figure 8. Noncooperative relaxation times calculated from eq 6, using $\mathrm{n}(=1-\beta)=0.49$ and 0.67 respectively for linear PDMS and the most-cross-linked network.

are related according to ${ }^{41}$

$$
\tau_{0} \sim \tau_{\alpha}(T)^{1-n}
$$

In the absence of inhomogeneous line broadening, the exponent $1-\mathrm{n}$ would equal the $\beta$ obtained from fitting eq 2 to the experimental dielectric loss spectrum. However, the values listed in Table 2 for the stretch exponent of the networks describe peaks whose shapes are affected by inhomogeneous broadening; that is, a distribution of environments exists, as governed by spatial or topological proximity of a segment to a network junction. Thus, while for linear PDMS, $\mathrm{n}=1$ $-\beta=0.49$, for the networks, the $\mathrm{n}$ in eq 6 only describes that component of the peak breadth resulting from intermolecular cooperativity. This means that $\mathrm{n}(<1-$ $\beta$ ) is treated as an adjustable parameter, in attempting to superimpose the $T_{\alpha}$-normal ized Arrhenius pl ots of $\tau_{0}$ for the most cross-linked network onto the curve for linear PDMS.

The results are shown in Figure 8. The effect of intermolecular constraints having been removed via eq 6 , the $\tau_{0}$-fragility curves approximately superimpose at lower temperatures. The value obtained for $\mathrm{n}$ for the PDMS network $\left(\mathrm{M}_{\mathrm{c}}=186 \mathrm{~g} / \mathrm{mol}\right)$ is 0.67 , reflecting a strong degree of intermolecular cooperativity. This implies that $\beta$ in the absence of inhomogeneous broadening would equal 0.33 . The full width at the halfintensity points (fwhm) of the Kohlrausch function for this value of $\beta$ is 3.28 decades, substantially less than the breadth observed in Figure 4 for this network. This is consistent with the idea that both enhanced intermolecular cooperativity and a distribution of environments contribute to the breadth of the observed $\alpha$-relaxation function for cross-linked polymers.

Previously, the crystallization behavior of very similar PDMS materials was investigated. ${ }^{43}$ The reduction of both the extent and thermodynamic stability of the crystalline phase was examined as a function of the degree of cross-linking of end-linked networks. From the variation of the equilibrium melting point, it was determined that a network junction precludes roughly eight adjoining chain units from incorporating into the crystal phase. This corresponds surprisingly well to the length scale determined herein for the $\alpha$-relaxation. This agreement may be somewhat fortuitous, although certainly the same local dynamics underlying the $\alpha$-relaxation are involved in crystal nucleation and growth. ${ }^{44}$

\section{Summary}

End-linked tetrafunctional poly(dimethylsiloxane) networks, spanning a broad range of cross-link densities, were prepared by the reaction of vinyl-terminated linear PDMS with tetrakis(dimethylsiloxy)silane, using a platinum-divinyltetramethyl disiloxane catalyst. The thermal expansivity, the dynamic glass temperature, the elastic modulus, and both the time and $\mathrm{T}_{\alpha}$-normalized temperature dependences of the local segmental relaxation were measured and found to be a function of crosslink density. Most significant was the marked change in the $\alpha$-relaxation properties when the network chain length was less than ca. $14 \AA$. This defines a characteristic length for the $\alpha$-relaxation, which is quite close to the value associated with suppression of the crystalline melting point and the degree of crystallinity in similar PDMS networks. It is also of the same magnitude as determinations of the length scale of the $\alpha$-relaxation in various other glass formers, both molecular and polymeric.

Acknowledgment. This work was supported by the Office of Naval Research. The authors thank Kelly M. Gray of the U.S. Naval Academy for experimental assistance.

\section{References and Notes}

(1) Dalnoki-Veress, E.; Forrest, J . A.; Murray, C.; Gigault, C.; Dutcher, J. R. Phys. Rev. E 2001, 63, 1801.

(2) Rizos, A. K.; Ngai, K. L. Phys. Rev. E 1999, 59, 612.

(3) Deschenes, L. A.; Vanden Bout, D. A. Science 2001, 292, 255.

(4) Schmidt-Rohr, K.; Spiess, H. W. Phys. Rev. Lett. 1991, 66, 3020.

(5) Ediger, M. D. Annu. Rev. Phys. Chem. 2000, 5199.

(6) Casalini, R.; Ngai, K. L.; Robertson, C. G.; Roland, C. M. J . Polym. Sci., Polym. Phys. 2000, 38, 1841.

(7) Leon, C.; Ngai, K. L.; Roland, C. M. J . Chem. Phys. 1999 110,11585

(8) Ngai, K. L.; Lunkenheimer, P.; Leon, C.; Schneider, U.; Brand, R.; Loidl, A. J . Chem. Phys. 2001, 115, 1405.

(9) Schneider, U.; Brand, R.; Lunkenheimer, P.; Loidl, A. Phys. Rev. Lett. 2000, 84, 5560 .

(10) Caliskan, G.; Kisliuk, A.; Novikov, V. N.; Sokolov, A. P. J . Chem. Phys. 2001, 114, 10189.

(11) Lunkenheimer, P.; Pimenov, A.; Loidl, A. Phys. Rev. Lett. 1997, 78, 2995.

(12) Casalini, R.; Ngai, K. L.; Roland, C. M. J . Chem. Phys. 2000, $112,5181$.

(13) Ngai, K. L.; Roland, C. M.; Yee, A. F. Rubber Chem. Technol. 1993, 66, 817.

(14) Roland, C. M. Macromolecules 1994, 27, 4242.

(15) Ngai, K. L.; Roland, C. M. Macromolecules 1994, 27, 2454.

(16) Roland, C. M.; Ngai, K. L.; Plazek, D. J . Comput. Theor. Polym. Sci. 1997, 7, 133.

(17) Suman, K. P.; Malone, S.; Cohen, C.; Gillmor, J . R.; Colby, R. H. Macromolecules 1992, 25, 5241.

(18) Llorente, M. A.; Mark, J . E. Macromolecules 1980, 13, 681.

(19) Valles, E. M.; Macosko, C. W. Macromol ecules 1979, 12, 673.

(20) Mott, P. H.; Roland, C. M. Macromolecules 1996, 29, 6941.

(21) Treloar, L. R. G. Rep. Prog. Phys. 1973, 36, 755.

(22) Kuo, A. C. M. In Polymer Data Handbook; Mark, J . E., Ed.; Oxford University Press: New York, 1999; p 411.

(23) Glatz-Reichenback, J . K. W.; Sorriero, L.; Fitzgerald, J . J . Macromolecules 1994, 27, 1338.

(24) Kohlrausch, R. Pogg. Ann. Phys. 1847, 12, 393. 
(25) Ferry, J . D. Viscoelastic Properties of Polymers; Wiley: New York, 1980.

(26) Roland, C. M.; Ngai, K. L. Macromolecules 1992, 25, 5765.

(27) Oldekop, V. W. Glastech. Ber. 1957, 30, 8.

(28) Laughlin, W. T.; Uhlmann, D. R. J . Phys. Chem. 1972, 76, 2317.

(29) Plazek, D. J .; Ngai, K. L. Macromolecules 1991, 24, 1222.

(30) Bohmer, R.; Ngai, K. L.; Angell, C. A.; Plazek, D. J . J . Chem. Phys. 1993, 99, 4201.

(31) Hodge, I. M. J . Non-Cryst. Solids 1996, 202, 164

(32) Roland, C. M.; Ngai, K. L. Macromolecules 1996, 29, 5747.

(33) Flory, P.J . Statistical Mechanics of Chain Molecules; Oxford University Press: New York, 1988.

(34) Ngai, K. L.; Roland, C. M. Macromolecules 1993, 26, 6824.

(35) Flory, P.J.; Semlyen, J . A. J . Am. Chem. Soc. 1966, 88, 3209

(36) Hempel, E.; Hempel, G.; Hensel, A.; Schick, C.; Donth, E. J. Phys. Chem. B 2000, 104, 2460.
(37) Reinsberg, S. A.; Qiu, X. H.; Wilhelm, M.; Spiess, H. W.; Ediger, M. D. J . Chem. Phys. 2001, 114, 7299.

(38) Tracht, U.; Wilhelm, M.; Heuer, A.; Spiess, H. W. J . Magn. Reson. 1999, 140, 460.

(39) Rizos, A. K.; Ngai, K. L. Phys. Rev. E 1999, 59, 612.

(40) Donth, E.; Huth, H.; Beiner, M. J . Phys.: Condens. Matter 2001, 13, L451.

(41) Ngai, K. L.; Rendell, R. W. J . Non-Cryst. Solids 1991, 131$133,942$.

(42) Ngai, K. L. J . Phys.: Condens. Matter 1999, 11, A119.

(43) Roland, C. M.; Aronson, C. A. Polym. Bull. (Berlin) 2000, 45, 439.

(44) Mankelkern, L. In Physical Properties of Polymers, 2nd ed.; Mark, J. E., Ed.; American Chemical Society: Washington, DC, 1993.

$\mathrm{MA011678H}$ 